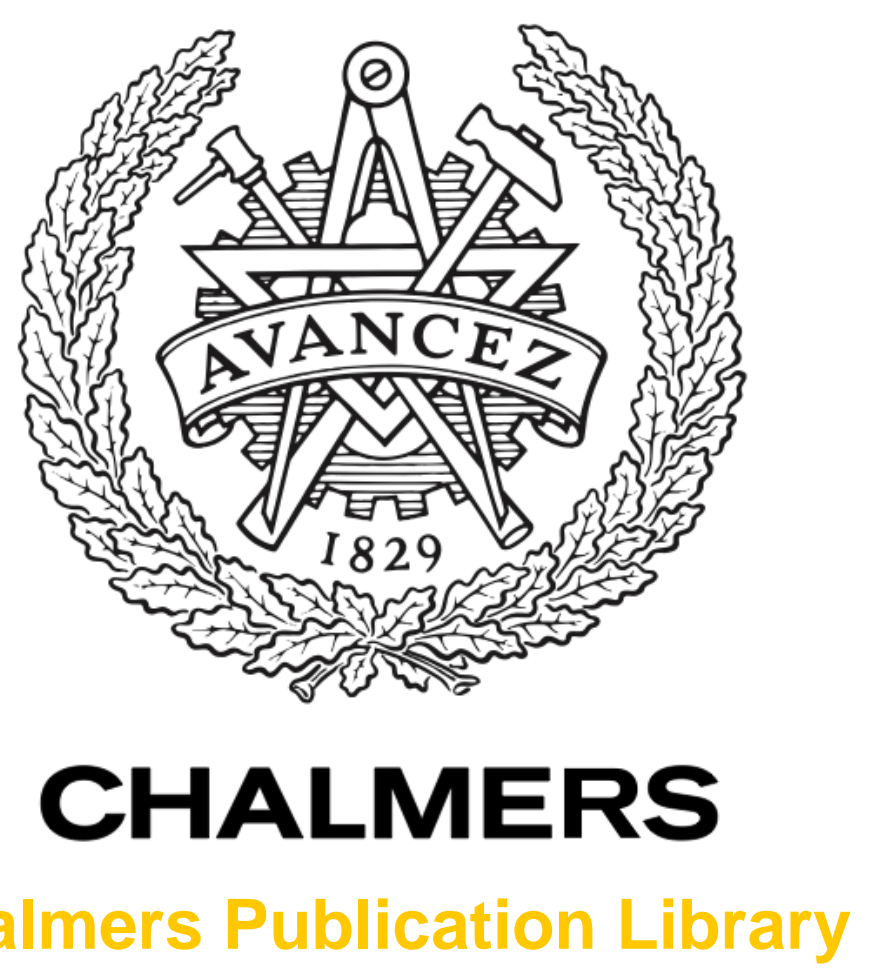

Chalmers Publication Library

\title{
ZnO/AIN Clad Waveguides for AlGaN-Based Quantum Cascade Lasers
}

This document has been downloaded from Chalmers Publication Library (CPL). It is the author's version of a work that was accepted for publication in:

Japanese Journal of Applied Physics (ISSN: 0021-4922)

Citation for the published paper:

Stattin, M. ; Bengtsson, J. ; Larsson, A. (2013) "ZnO/AlN Clad Waveguides for AlGaNBased Quantum Cascade Lasers". Japanese Journal of Applied Physics, vol. 52(5), pp. 054001 .

http://dx.doi.org/10.7567/JJAP.52.054001

Downloaded from: http://publications.lib.chalmers.se/publication/176084

Notice: Changes introduced as a result of publishing processes such as copy-editing and formatting may not be reflected in this document. For a definitive version of this work, please refer to the published source. Please note that access to the published version might require a subscription. 


\title{
ZnO/AlN Clad Waveguides for AlGaN-Based Quantum Cascade Lasers
}

\author{
Martin Stattin*, Jörgen Bengtsson, and Anders Larsson \\ Photonics Laboratory, Department of Microtechnology and Nanoscience, \\ Chalmers University of Technology, SE-41296 Göteborg, Sweden
}

\begin{abstract}
Waveguide designs for AlGaN-based near-infrared quantum cascade lasers are proposed and analyzed using optical mode solvers. Because of the poor electrical conductivity, AlN is not a suitable cladding material for the $\mathrm{AlN} / \mathrm{GaN} / \mathrm{AlGaN}$ gain region. Instead we propose the use of $\mathrm{ZnO}$ as a conductive top cladding and to embed the gain region between AlGaN current injection and extraction regions so that an AlN substrate can be used as the lower cladding. We also examine the use of an AlN template on a SiC substrate and find that it can also provide sufficient mode confinement with negligible radiation loss into the SiC substrate. We identify a single mode ridge waveguide design with reasonable mode confinement $(40 \%)$ and loss $\left(39 \mathrm{~cm}^{-1}\right)$.
\end{abstract}

\section{Introduction}

The quantum cascade laser (QCL) has since its first demonstration $^{1)}$ become the semiconductor laser source of choice in the mid and long wavelength infrared. The most efficient QCLs are InGaAs/AlInAs/InP-based and operate at wavelengths between 4 and $9 \mu \mathrm{m} .{ }^{2}$ ) Longer wavelengths can be reached using the AlGaAs/GaAs material system, with QCLs operating in the 1-5 $\mathrm{THz}$ range being demonstrated, ${ }^{3)}$ although not at room temperature. For wavelengths shorter than $4 \mu \mathrm{m}$, the conduction band offset between InGaAs and AlInAs becomes a limiting factor. Therefore, other semiconductors with larger offset such as InAs/AlSb have been used to reach down to $2.63 \mu \mathrm{m} .^{4)}$

For emission at even shorter wavelengths, yet another material system is likely required. Currently, Al$\mathrm{GaN}$ is considered to be a promising system. This is mainly due to the large AlN/GaN conduction band offset, large remote-valley separation and wide optical transparency. ${ }^{5)}$ Even operation at telecom wavelengths is theoretically possible, ${ }^{6)}$ with potentially temperature stable operation ${ }^{7)}$ and low chirp.

Early studies show intersubband (ISB) absorption ranging between 1.75-4.2 $\mu \mathrm{m}$ from GaN/AlGaN quantum wells (QWs). $\left.{ }^{8}, 9\right)$ Using GaN/AlN (QWs), ISB detection has been demonstrated at $\left.1.55 \mu \mathrm{m} .{ }^{10}\right)$ Optically pumped ISB emission from GaN/AIN QWs at $2.13 \mu \mathrm{m}^{11}$ ) has been demonstrated as well as optically pumped ISB emission at $1.5 \mathrm{\mu m}$ from a stack of $\mathrm{AlN} / \mathrm{GaN}$ quantum dots (QDs). ${ }^{12)}$ However, electrically pumped near infrared (NIR) ISB emission has not yet been demonstrated in the AlGaN system. This can partially be attributed to the short nonradiative lifetime of the upper energy state causing a low spontaneous emission rate for ISB transitions. Less than one in a million of the electrons in the upper state generate spontaneous emission, ${ }^{13,14)}$ potentially making lasing through stimulated (amplified) emission easier to demonstrate than spontaneous emission.

For the realization of AlGaN-based NIR QCLs, apart from the challenging design of the active region, lowloss optical waveguides that allow both optical confinement and current injection are needed. The design of

\footnotetext{
*E-mail: martin.stattin@chalmers.se
}

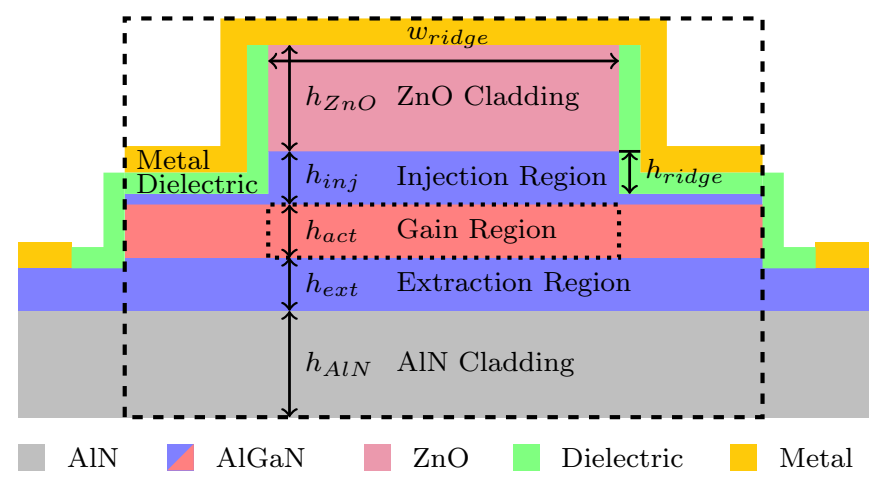

Fig. 1. (Color online) The proposed waveguide design. The dashed box indicates the numerical window used for designing and analyzing the single mode waveguide while the dotted area defines the region used for calculating the optical confinement factor.

such waveguides is not a straightforward task since the use of low refractive index n-type AlN as cladding layers (surrounding the core with active $\mathrm{AlN} / \mathrm{GaN}$ gain stages) is not possible due to the low doping efficiency of AlN, and consequently large electrical resistivity, $\geq 1 \Omega \cdot \mathrm{cm} .{ }^{15)}$ With higher conductivity n-type AlGaN as cladding layers, the refractive index difference between core and cladding becomes insufficient for vertical confinement of the optical field which may lead to strong interaction with metal contacts, and therefore high optical loss, in conventional waveguide structures (e.g., a ridge waveguide). ${ }^{16)}$

There are experimental demonstrations of AlGaNbased waveguides for optical switching ${ }^{17)}$ and ISB absorption $^{5,18,19)}$ at NIR wavelengths. However, the absorption loss caused by metal contacts was not taken into consideration. For current driven QCLs it is essential to minimize such optical loss while keeping the electrical resistance low. A waveguide with a GaN core and lattice matched InAlN cladding layers is proposed in Ref. 20 and a TM loss of $11 \mathrm{~cm}^{-1}$ is demonstrated at $1.55 \mu \mathrm{m}$ for a passive waveguide. Unfortunately, when increasing the $\mathrm{Al}$ mole fraction in the core, as required in QCLs, the reduced refractive index contrast necessitates an increased $\mathrm{Al}$ fraction in the cladding layers potentially reducing their conductivity. In Ref. 21 an ISB electro-absorption modulator design was pro- 
posed, utilizing electron-plasma effects in heavily doped $\left(10^{20} \mathrm{~cm}^{-3}\right)$ GaN cladding layers for confinement. However, the optical loss with this approach is likely too large for QCLs since the calculated modal loss is $230 \mathrm{~cm}^{-1}{ }^{21}$ ) In Ref. 16 we show that by using an off-center contact geometry, waveguides with metal induced losses below $10 \mathrm{~cm}^{-1}$ are possible. However, the off-center geometry may lead to non-uniform current injection and thereby reduced electrical injection efficiency. Also, the proposed use of direct growth on sapphire will make growth of high quality QWs difficult.

To alleviate these problems we propose an improved waveguide design utilizing n-type $\mathrm{ZnO}$ as a transparent and conductive upper cladding layer and an AlN substrate, or an AlN layer on a SiC substrate, as the lower cladding layer (Fig. 1). The design is evaluated at $1.55 \mathrm{\mu m}$, the preferred wavelength for low loss transmission on optical fiber, but is not fundamentally limited to this wavelength.

\section{Waveguide Design}

Initially, we consider the vertical waveguide design. As the upper cladding layer we use n-type $\mathrm{ZnO}$, which has a lower refractive index than AlN, a high electrical conductivity $^{22)}$ and a similar lattice constant. ${ }^{23)}$ Other oxides, such as $\mathrm{MgZnO}$, have similar properties and even lower refractive index ${ }^{24)}$ and can also be used. However, $\mathrm{ZnO}$ is likely a better choice due to higher material quality and thermal conductivity.

Between the $\mathrm{n}-\mathrm{ZnO}$ cladding layer and the gain region, an n-AlGaN current injection layer is used for injection of electrons into the gain region. The gain region consists of a stack of gain stages, each containing several GaN QWs and AlN barriers. Interlacing the gain stages are thin AlGaN transport layers that match the injection and extraction energies between adjacent gain stages. ${ }^{1,16)}$ For the optical simulations we model the gain region as a bulk AlGaN layer with the same composition as the average of the gain region. This reduces the modeling complexity and is not believed to introduce significant errors, considering the large difference between the optical wavelength $(1.55 \mu \mathrm{m})$ and the thickness of the gain stages $(\approx 5 \mathrm{~nm})$ and transport layers $(\approx 10 \mathrm{~nm})$. Below the gain region, an n-AlGaN current extraction region is used for extraction of electrons from the gain region. The gain region, together with the injection and extraction layers, forms the core of the waveguide. The injection and the extraction regions both have the same AlGaN composition as the average composition of the gain region. The relative thickness of injection and extraction regions can be adjusted to center the maximum of the mode intensity in the gain region.

To optically confine the mode from below we use a substrate with lower refractive index than that of the waveguide core. Such substrates are AlN, sapphire and $\mathrm{ZnO}$. AlN substrates are not as mature as sapphire and $\mathrm{ZnO}$ substrates, but the quality of the epitaxial structure is expected to be significantly improved when grown on an AlN substrate. SiC substrates are available in large dimensions and can be used for the growth of high quality AlGaN epitaxial layers; as an example continuous
Table I. Refractive indices at $1.55 \mu \mathrm{m}$ for the materials used in the simulations.

\begin{tabular}{llll}
\hline Material & $n_{o}$ & $n_{e}$ & Reference \\
\hline $\mathrm{GaN}$ & 2.2873 & 2.3160 & 27 \\
$\mathrm{Al}_{0.25} \mathrm{Ga}_{0.75} \mathrm{~N}$ & 2.2247 & 2.2552 & \\
$\mathrm{Al}_{0.5} \mathrm{Ga}_{0.5} \mathrm{~N}$ & 2.1620 & 2.1945 & \\
$\mathrm{Al}_{0.75} \mathrm{Ga}_{0.25} \mathrm{~N}$ & 2.0994 & 2.1337 & \\
$\mathrm{AlN}$ & 2.0367 & 2.0729 & 27 \\
$\mathrm{SiC}$ & 2.5752 & 2.6197 & 28 \\
$\mathrm{ZnO}$ & 1.9280 & 1.9381 & 24 \\
$\mathrm{SiO}_{2}$ & 1.5 & - & - \\
\hline
\end{tabular}

lasing at $348 \mathrm{~nm}$ has been demonstrated ${ }^{25)}$ using such substrates. Unfortunately, the refractive index of $\mathrm{SiC}$ at $1.55 \mathrm{\mu m}$ is much higher than that of AlGaN and can therefore not be used for mode confinement. However, a thick AlN layer grown on a $\mathrm{SiC}$ substrate (a template) can potentially be used as the lower cladding layer. The material quality achieved with this approach is expected to be significantly better than that of growth on sapphire. We therefore examine the extra optical loss introduced when using an AlN layer on a SiC substrate (optical leakage into the high index substrate) and compare with the use of an AlN substrate.

Lateral mode confinement is achieved using a ridge waveguide covered by a dielectric material, with the strength of the confinement given by the height and width of the ridge. An opening in the dielectric enables the formation of a metal contact for vertical current injection through the $\mathrm{n}-\mathrm{ZnO}$ cladding layer and the $\mathrm{n}-\mathrm{AlGaN}$ injection region into the gain region. Extraction of current occurs through the n-AlGaN extraction layer and subsequent lateral transport to metal contacts placed on both sides of the ridge.

\section{Simulations}

To model specific optical loss mechanisms in the waveguide, and their dependencies on design and layer thicknesses, we use one-dimensional (1D) (slab waveguide) simulations for different vertical cross-sections of the waveguide (Fig. 2). Both FIMMWAVE from Photon Design and COMSOL Multiphysics 3.5a are used in the simulations. A 2D waveguide model is used to find a single mode ridge waveguide design (Fig. 1) and to examine the properties of the waveguide.

Due to the selection rules for the ISB transitions, optical fields with the Electrical-field component perpendicular to the plane of the QWs are preferentially amplified. ${ }^{26)}$ The optical fields are therefore TM-polarized and boundary conditions are set accordingly. All materials used in the waveguide, except for dielectric $\left(\mathrm{SiO}_{2}\right)$ and metal $(\mathrm{Au})$, are birefringent. With growth on c-plane substrates, the refractive index experienced by TE and TM polarized modes is the ordinary $\left(n_{o}\right)$ and the extraordinary $\left(n_{e}\right)$, respectively. In the $1 \mathrm{D}$ geometries, the extraordinary refractive index was therefore selected, while in the $2 \mathrm{D}$ simulations a refractive index tensor was used for the birefringent materials. The refractive indices used are listed in Table I. 
For AlN and GaN we use the refractive index model in Ref. 27. Linear interpolation is used for the AlGaN alloys. For $\mathrm{ZnO}$ we use the Sellmeier equation and parameters from Ref. 24 . In ref. 28, a relation quantifying the refractive index of $4 \mathrm{H}-\mathrm{SiC}$ at wavelengths from 0.467 to $0.691 \mu \mathrm{m}$ is presented. We assume this can be extrapolated to $1.55 \mathrm{\mu m}$ and we validated by comparing the ordinary part with Ref. 29 in the NIR. For $\mathrm{SiO}_{2}$, we use a refractive index of 1.5 .

The metal contact is modeled as a layer of gold. Although this is not the actual ohmic contact metallization it gives enough absorption to determine whether optical energy is absorbed by the contact or not. For gold we use the complex refractive index $0.56+\mathrm{i} 10.8 .{ }^{30}$ )

Depending on the design of the gain region, the average AlGaN composition differs. We selected three compositions that encompass most of the solutions, $\mathrm{Al}_{0.25} \mathrm{Ga}_{0.75} \mathrm{~N}, \mathrm{Al}_{0.5} \mathrm{Ga}_{0.5} \mathrm{~N}$, and $\mathrm{Al}_{0.75} \mathrm{Ga}_{0.25} \mathrm{~N}$. Since the injection and extraction regions are chosen to have the same material composition as the average of the gain region, we treat all three regions as a single layer (the core of the waveguide) with the height $h_{\text {core }}=$ $h_{i n j}+h_{a c t}+h_{\text {ext }}$ (Fig. 2) in the 1D simulations.

When using an AlN layer on a $\mathrm{SiC}$ substrate, instead of a bulk AlN substrate, the tail of the guided modes extends into the $\mathrm{SiC}$, causing radiation leakage. To model the leakage loss, we use a perfectly matched layer (PML) as a lower boundary. The PML solution is implemented using COMSOL Multiphysics 3.5a. In the model [Fig. $2(\mathrm{a})]$, the waveguide core was placed between the $\mathrm{ZnO}$ upper cladding layer and the AlN lower cladding layer. Beneath the AlN layer, we placed $2 \mu \mathrm{m} \mathrm{SiC}$ and used a $2 \mu \mathrm{m}$ PML as a lower boundary. To isolate the leakage loss, all layers were non-absorbing. The only loss mechanism is then radiation into the PML and we obtain the leakage loss. We varied the thickness of the AlN layer, $h_{A l N}$, and the thickness of the core, $h_{\text {core }}$, to investigate their effects on the radiation loss.

The purpose of the $\mathrm{n}-\mathrm{ZnO}$ cladding layer is to provide current transport while shielding the optical mode from the metal contact. High electrical conductivity and low optical loss are important characteristics of this layer. According to Ref. 22, the absorption in $\mathrm{n}-\mathrm{ZnO}$ at $1.55 \mu \mathrm{m}$ is dominated by free carrier absorption (FCA). We use a FCA coefficient of $200 \mathrm{~cm}^{-1}$, corresponding to a free electron density of $1 \cdot 10^{19} \mathrm{~cm}^{-3}$. $\left.^{22}\right)$ The effective index solver in FIMMWAVE is used to examine the effect of varying the thickness of the $\mathrm{ZnO}$ cladding layer, $h_{Z n O}$, while keeping the thickness of the waveguide core, $h_{\text {core }}$, fixed and vice versa. Only losses in $\mathrm{ZnO}$ cladding and metal contact were included. The refractive index profile used in the simulation is that of Fig. 2(b).

After having determined suitable design parameters via $1 \mathrm{D}$ simulations a $2 \mathrm{D}$ model was implemented in the finite element solver in FIMMWAVE and used to design a single mode ridge waveguide. The numerical window is indicated by the dashed box in Fig. 1. In this simulation an AlN substrate was used as the lower cladding. The core height, $h_{\text {core }}$, was set to $1.5 \mu \mathrm{m}$, divided equally between $h_{i n j}, h_{a c t}$, and $h_{\text {ext }}$. The thickness of the dielectric $\left(\mathrm{SiO}_{2}\right)$ layer used to isolate the sides of the ridge from

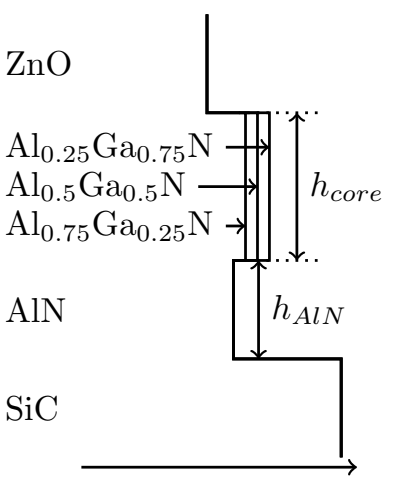

ref. ind.

(a)

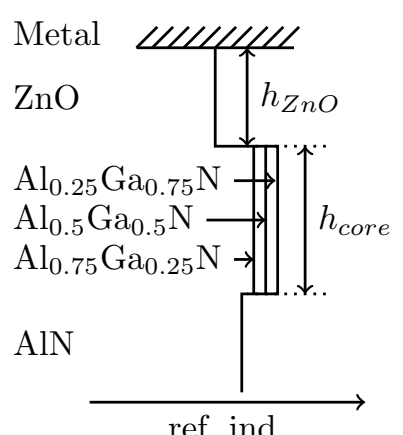

(b)
Fig. 2. Refractive index profiles used to investigate leakage and absorption loss. With (a) we model the leakage loss into the $\mathrm{SiC}$ substrate. With (b) we examine the absorption loss in the $\mathrm{ZnO}$ cladding and in the metal contact.

the contact metal was set to $200 \mathrm{~nm}$. The $\mathrm{ZnO}$ layer thickness was fixed at $1 \mu \mathrm{m}$ and the FCA coefficient at $200 \mathrm{~cm}^{-1}$. For the FCA coefficient in the AlGaN injection, gain, and extraction regions we use FCA data for n-doped $\mathrm{GaN}^{31,32)}$ and assume similar values for $\mathrm{FCA}$ in AlGaN. Based on this, we use a FCA coefficient of $50 \mathrm{~cm}^{-1}$ in the injection and extraction regions corresponding to a free carrier concentration of $7 \times 10^{17} \mathrm{~cm}^{-3}$. For the gain region the FCA coefficient depends strongly on how the QCL stack is designed. Due to the large discrepancies between available designs ${ }^{6,16)}$ we assume half the gain region to consist of doped layers, corresponding to partially doped transport layers, giving an average FCA coefficient of $25 \mathrm{~cm}^{-1}$. Additionally, we examine the case of a lossless gain region. Finally, the width of the ridge was varied between 2 and $5 \mu \mathrm{m}$ and the etch depth into the injection layer $\left(h_{\text {ridge }}\right.$, Fig. 1$)$ between 0 and $0.5 \mu \mathrm{m}$.

The confinement factor was calculated as the mode overlap with the area within the dotted region in Fig. 1. Thus we assume that only the gain region immediately beneath the ridge will amplify the modes supported by the waveguide. This implies that lateral diffusion of electrons in the gain region is assumed to be negligible. The two TM polarized modes with the highest confinement factors were analyzed.

\section{Results}

From the analysis of required AlN cladding layer thickness, $h_{A l N}$, on $\mathrm{SiC}$, we found an exponential reduction of the radiation leakage loss with increasing $h_{A l N}$ [Fig. 3(a)]. The decay factor depends on the dimensions of the waveguide. Using a waveguide core thickness $h_{\text {core }}=1.5 \mathrm{\mu m}$, the leakage loss is below $1 \mathrm{~cm}^{-1}$ for all the core AlGaN compositions studied when $h_{A l N}>$ $2.3 \mathrm{\mu m}$. In Fig. 3(b) we also show the dependence of leakage loss on core thickness at a fixed AlN cladding thickness.

From the analysis of required thickness of the $\mathrm{ZnO}$ cladding layer, $h_{Z n O}$, we found an initial reduction and a subsequent saturation of the modal absorption loss with 


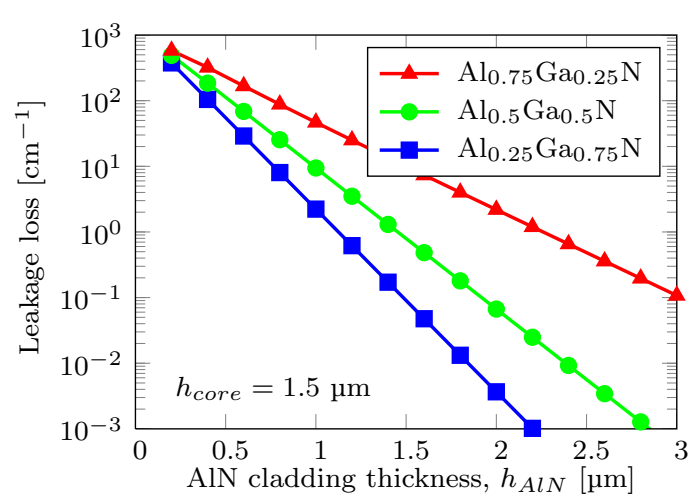

(a)

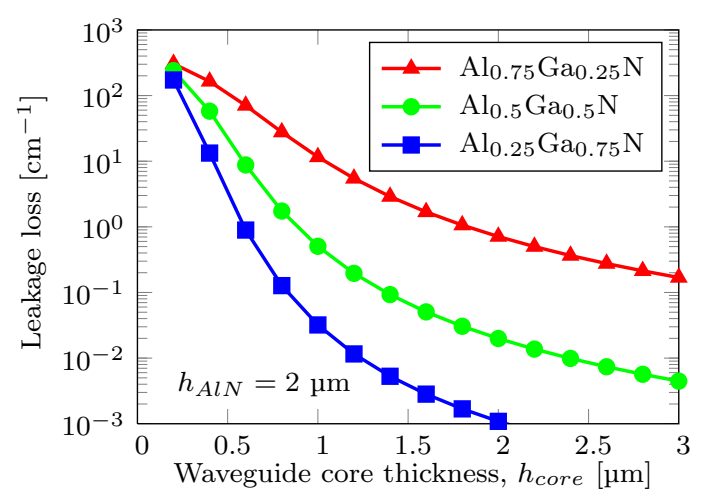

(b)

Fig. 3. (Color online) Leakage loss into $\mathrm{SiC}$ substrate as function of (a) the AlN cladding thickness, $h_{A l N}$, with waveguide core thickness $h_{\text {core }}=1.5 \mu \mathrm{m}$, and (b) the waveguide core thickness, $h_{\text {core }}$, with AlN cladding thickness $h_{A l N}=2 \mu \mathrm{m}$.

increasing thickness. The saturation value and the onset of saturation depend on both the FCA coefficient in the $\mathrm{ZnO}$ layer and on the core thickness and AlGaN composition. In Fig. 4(a) it is seen that for a core thickness of $1.5 \mu \mathrm{m}$, a $1 \mu \mathrm{m}$ thick $\mathrm{ZnO}$ layer is sufficient to reach saturation and thus isolate the optical mode from the metal contact. In Fig. 4(b) we show how the absorption decreases with increasing core thickness at a $\mathrm{ZnO}$ thickness of $1 \mu \mathrm{m}$. Due to the non-symmetric refractive index profile, guided modes could not be found for all AlGaN compositions and core thicknesses.

The 2D waveguide model is used to identify the single mode design window. Since higher order modes are always found due to the boundaries of the simulation window we need to select a cut-off confinement factor for which it can be assumed that the higher order mode is either a false solution or is very weakly amplified with respect to the fundamental mode. In Fig. 5 we plot the confinement factor of the two TM polarized modes with the highest confinement factors for the three core AlGaN compositions examined. We observe that when the higher order mode appears it has a confinement factor somewhere between 0.1 and 0.15 . We therefore assume that waveguide designs with a confinement factor lower than 0.15 for the higher order mode are effectively single mode. The modal loss for the fundamental mode of all these waveguides varies between 33 and $41 \mathrm{~cm}^{-1}$. In the case of a lossless gain region (with loss from injection,

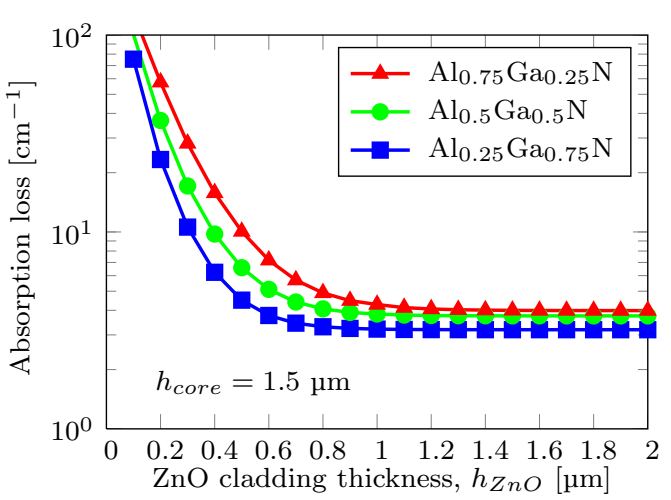

(a)

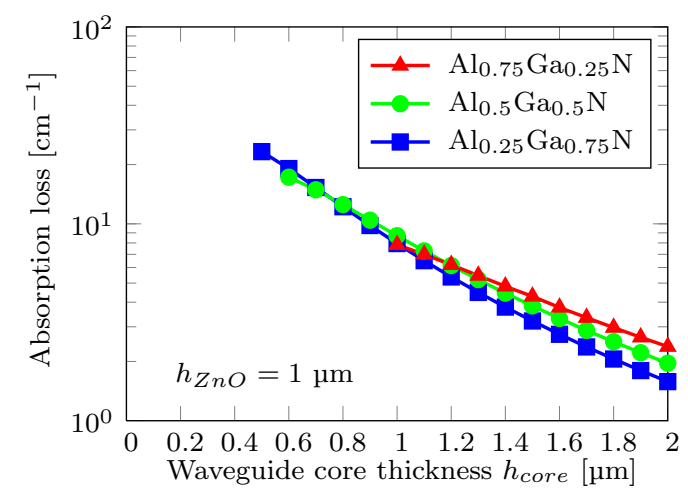

(b)

Fig. 4. (Color online) Combined absorption loss in the $\mathrm{ZnO}$ cladding and in the contact metallization as a function of (a) the $\mathrm{ZnO}$ cladding thickness, $h_{Z n O}$, with waveguide core thickness $h_{\text {core }}=1.5 \mu \mathrm{m}$ and (b) the waveguide core thickness, $h_{\text {core }}$, with $\mathrm{ZnO}$ cladding thickness $h_{Z n O}=1 \mu \mathrm{m}$.

extraction, cladding and contact layers) the modal loss reduces by approximately $10 \mathrm{~cm}^{-1}$, depending on modal overlap with the gain region.

\section{Discussion}

To further analyze the waveguide properties we select a single mode design with a mean core composition of $\mathrm{Al}_{0.5} \mathrm{Ga}_{0.5} \mathrm{~N}$. The ridge is $3 \mu \mathrm{m}$ wide and is etched 0.4 $\mu \mathrm{m}$ into the injection region. The cross-sectional intensity distribution of the fundamental mode is shown in Fig. 6 . The confinement factor is 0.40 and the modal loss is 39 $\mathrm{cm}^{-1}$, while the confinement factor for the higher order mode is 0.12 with tails extending to the boundaries of the simulation window. The mode index for the fundamental mode is $n_{\text {eff }}=2.15$ and the far-field angular full-width at half-maximum of the beam emitted through a cleaved facet is $38^{\circ}$ in the vertical plane and $18^{\circ}$ in the horizontal plane. Most of the modal loss is due to FCA in the doped AlGaN layers. In agreement with Fig. 4(a), less than 4 $\mathrm{cm}^{-1}$ of the absorption occurs in the $\mathrm{ZnO}$ cladding and metal contact.

Additional loss due to scattering caused by sidewall roughness (at the $\mathrm{AlGaN} / \mathrm{SiO}_{2}$ interface) created during the dry etching of the waveguide is also to be expected. Considering results from numerical modeling of scattering loss in waveguides with a similar index contrast, ${ }^{34)}$ and an experimentally determined loss value of $11 \mathrm{~cm}^{-1}$ 
Fundamental TM mode Second TM mode

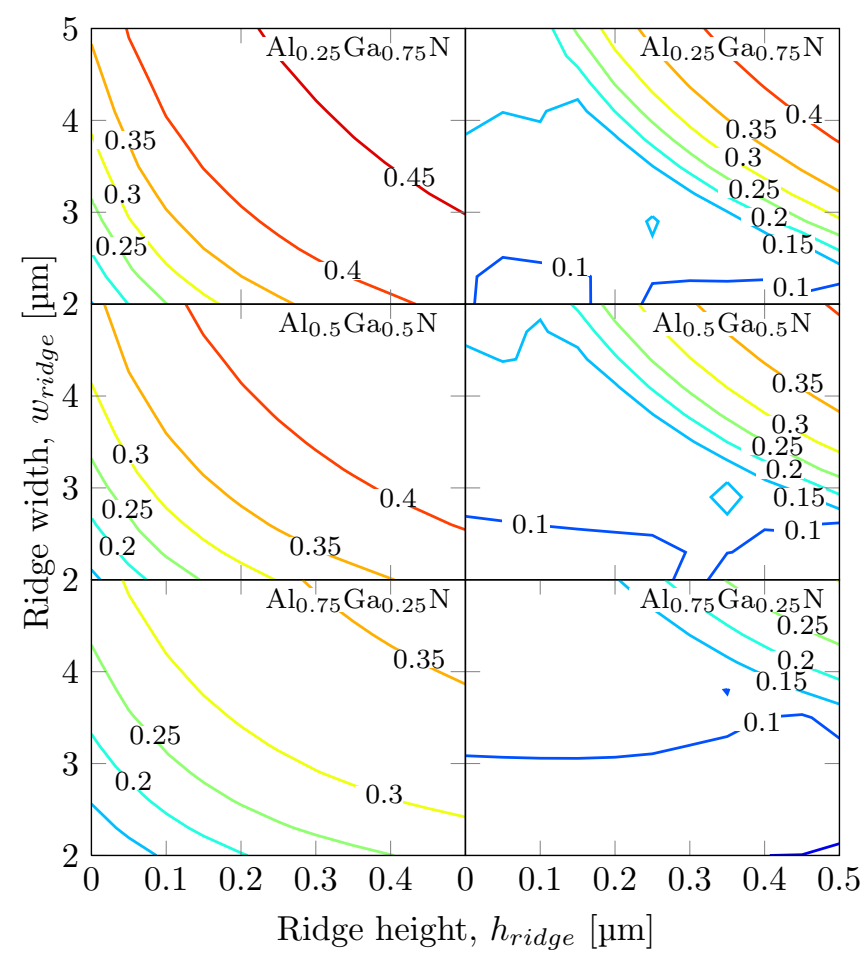

Fig. 5. (Color online) Contour plots of the confinement factor for the two lowest order TM modes when varying the width and height of the ridge for the three examined AlGaN compositions.

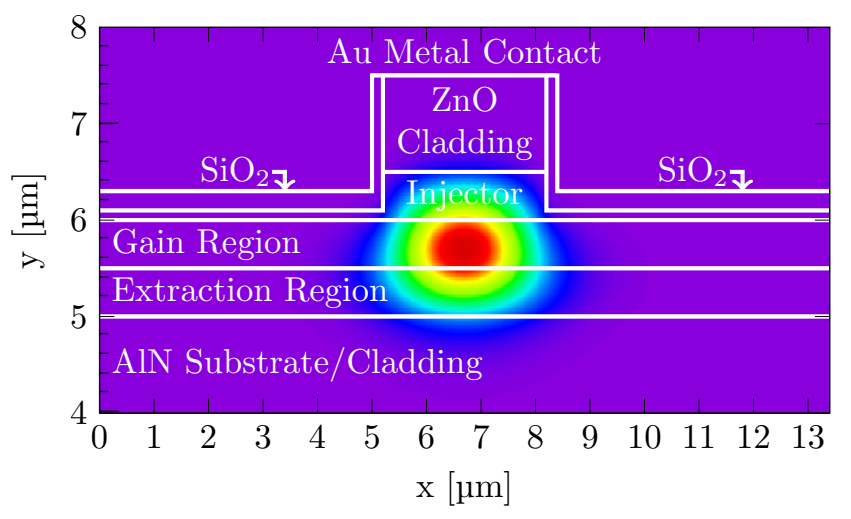

Fig. 6. (Color online) Mode profile of the fundamental mode for a waveguide with an $\mathrm{Al}_{0.5} \mathrm{Ga}_{0.5} \mathrm{~N}$ core and $w_{\text {ridge }}=3 \mu \mathrm{m}$ and $h_{\text {ridge }}=0.4 \mu \mathrm{m}$.

for a deep etched InAlN waveguide with a high index contrast air cladding, ${ }^{20}$ ) we expect to be able to reach a scattering loss value of only a few $\mathrm{cm}^{-1}$ under etching conditions optimized for smooth sidewalls.

To quantify the threshold gain of a QCL employing such a waveguide, the mirror transmission losses also have to be accounted for. With cleaved facets we estimate a power reflection of 0.13 , which for a $1 \mathrm{~mm}$ long QCL gives an additional loss of $20 \mathrm{~cm}^{-1}$. This can be reduced by depositing high reflectivity coatings on the facets or by using a distributed feedback structure (e.g., a waveguide with corrugated side-walls ${ }^{33)}$ ). The latter also enables the single longitudinal mode (single wavelength) operation needed in many applications.
With such techniques the modal gain at threshold (which balances the modal loss) is estimated at $\alpha_{\text {tot }} \approx 50$ $\mathrm{cm}^{-1}$. This requires a gain in the gain region $>100 \mathrm{~cm}^{-1}$ (with a confinement factor of $\Gamma=0.40$ ). To estimate the threshold current density, $J_{t h}$, we use: ${ }^{35)}$

$$
J_{t h}=\frac{\alpha_{t o t}}{g \Gamma}
$$

where the gain coefficient

$$
g=\tau_{3}\left(1-\frac{\tau_{2}}{\tau_{32}}\right) \frac{4 \pi e z_{32}^{2}}{\lambda \epsilon_{0} n_{e f f} L_{p} 2 \gamma_{32}}
$$

In Eg. (2) appears parameters representing the states and transitions involved in the stimulated emission process (where subscript 3 refers to the upper state and subscript 2 to the lower state), for which we need to use reasonable values. Based on measurements of ISB emission at $2 \mu \mathrm{m}$ from an AlN/GaN superlattice ${ }^{11,14)}$ we estimate the emission linewidth $2 \gamma_{32}=100 \mathrm{meV}$. We assume an upper state lifetime $\tau_{3}=400 \mathrm{fs}$, based on measurements of ISB absorption at $1.45 \mu \mathrm{m}$ in GaN/AlN QWs reported in. ${ }^{36)}$ Assuming phonon assisted emptying of the lower state we set the lifetime in this state to $\tau_{2}=100 \mathrm{fs}$. For the upper-to-lower state transition lifetime we use $\tau_{32}=600 \mathrm{fs}$. Finally, we set the length of one gain stage (the period of the QCL stack) to $L_{p}=15 \mathrm{~nm}$ and the matrix element to $z_{32}=1 \mathrm{~nm}$. This is all based on comparisons with conventional QCLs. ${ }^{35)}$ We then obtain a threshold current density of $13 \mathrm{kA} / \mathrm{cm}^{2}$, corresponding to a threshold current of $0.4 \mathrm{~A}$ for our example device. With this relatively large current density room temperature continuous lasing is challenging but the demonstration of lasing under pulsed conditions would still provide valuable results for further analysis and design improvements.

The results obtained from the $1 \mathrm{D}$ simulations show that all loss values can be reduced to low or insignificant values compared to the FCA loss in the core region (injection/extraction layers and gain region). The FCA depends on the free carrier density which, together with the carrier mobility, also determines the resistance in these regions.

To estimate the QCL resistance we treat it as a series connection of resistances representing the $\mathrm{ZnO}$ cladding layer, the AlGaN injection layer, QCL stack, and the AlGaN extraction layer. In the extraction layer, current is transported laterally to the contacts on both sides of the ridge. The contacts are assumed to be at a distance of 5 $\mu \mathrm{m}$ from the ridge. For the $\mathrm{ZnO}$ cladding layer, which is assumed to have a free electron density of $1 \times 10^{19} \mathrm{~cm}^{-3}$, we use a worst case mobility of $10 \mathrm{~cm}^{2} \cdot \mathrm{V}^{-1} \cdot \mathrm{s}^{-1},{ }^{22)}$ yielding a resistivity of $0.06 \Omega \cdot \mathrm{cm}$. For the AlGaN injection and extraction layers, which are assumed to have a free electron density of $7 \times 10^{17} \mathrm{~cm}^{-3}$, we use mobility from ${ }^{37}$ ) and obtain a resistivity of $0.44 \Omega \cdot \mathrm{cm}$. We assume parasitic resistivity in the QCL stack to be the same as in the injection layer. A QCL having a $3 \mu \mathrm{m}$ wide ridge and a $1 \mathrm{~mm}$ long cavity would then have a total resistance of $\approx 23 \Omega$, with $0.2 \Omega$ coming from the $\mathrm{ZnO}$ cladding layer, $0.7 \Omega$ from the injection layer, $0.7 \Omega$ from the QCL stack, 
and $22 \Omega$ from the extraction layer. Clearly, lateral transport along the $0.5 \mu \mathrm{m}$ thick extraction layer constitutes the largest contribution to the resistance. Increasing the carrier concentration in the extraction layer to $7 \times 10^{18}$ $\mathrm{cm}^{-3}$ would reduce the resistance in this layer to $2.2 \Omega$ and increase the FCA coefficient to $122 \mathrm{~cm}^{-1}$, resulting in a modal loss of $62 \mathrm{~cm}^{-1}$.

The analysis above does not account for additional resistance due to effects of electron transport across the $\mathrm{ZnO} / \mathrm{AlGaN}$ interface, where the conduction band offset varies between $0.7 \mathrm{eV}(\mathrm{ZnO} / \mathrm{GaN})^{38)}$ and $3 \mathrm{eV}$ $(\mathrm{ZnO} / \mathrm{AlN}) .{ }^{39)}$ Measurements of the resistivity of n-type $\mathrm{ZnO} / \mathrm{GaN}^{40)}$ and $\mathrm{ZnO} / \mathrm{Al}_{0.15} \mathrm{Ga}_{0.85} \mathrm{~N}$ interfaces ${ }^{41)}$ suggest that a resistivity as low as $1 \times 10^{-4} \Omega \cdot \mathrm{cm}^{2}$ can be achieved, which would translate into an additional resistance of only a few ohms. The insertion of highly doped thin layers at the interface may enable an even lower resistivity, although at the expense of a somewhat higher FCA loss.

To simplify fabrication, the $\mathrm{ZnO}$ cladding thickness can be reduced in the selected design. Reducing the thickness from 1.0 to $0.5 \mu \mathrm{m}$ gives a slight increase of the modal loss, from 39 to $42 \mathrm{~cm}^{-1}$. This is due to absorption in the upper metal contact. This is an increase of $3 \mathrm{~cm}^{-1}$ which is in good agreement with the results from the 1D simulation [Fig. 4(a)].

We also notice from Fig. 6 that the mode energy is not perfectly centered over the gain region. This is caused by the larger refractive index difference between core and $\mathrm{ZnO}$ cladding than between core and AlN cladding. The effect is enhanced by the ridge which pushes the mode towards the AlN cladding layer. This can be alleviated by, for instance, increasing the thickness of the current injection layer or reducing the thickness of the current extraction layer. The mode confinement can also be increased by replacing all or parts of the current injection region with stacks of gain stages, thereby extending the active region. It is also possible to simply increase the active region thickness to improve confinement and reduce losses. With a $1 \mu \mathrm{m}$ thick active region the confinement factor increases to 0.55 and the loss is reduced to $33 \mathrm{~cm}^{-1}$.

\section{Conclusions}

We have proposed a new waveguide design strategy for $1.55 \mu \mathrm{m}$ AlGaN-based QCLs, employing a ZnO upper cladding layer and an AIN lower cladding layer. Through simulations we have shown that the waveguide can provide sufficient optical confinement, with FCA in the doped layers being the dominant optical loss mechanism. A modal propagation loss of $39 \mathrm{~cm}^{-1}$ is obtained for a single mode waveguide on an AlN substrate and an additional loss of $1 \mathrm{~cm}^{-1}$ is expected if a $1.5 \mu \mathrm{m}$ thick AlN cladding layer on a $\mathrm{SiC}$ substrate is used instead. We have also suggested ways to modify the design to improve mode confinement, reduce optical loss, and reduce resistance.

\section{Acknowledgment}

We are grateful to K. Berland for helpful discussions on QCL gain structures.
1) J. Faist, F. Capasso, D. L. Sivco, C. Sirtori, A. L. Hutchinson, and A. Y. Cho: Science 264 (1994) 553.

2) M. Razeghi: IEEE J. Sel. Top. Quantum Electron. 5 (2009) 941.

3) S. Kumar, and A. Lee: IEEE J. Sel. Top. Quantum Electron. 14 (2008) 333.

4) O. Cathabard, R. Teissier, J. Devenson, J. C. Moreno, and A. N. Baranov: Appl. Phys. Lett. 96 (2010) 141110.

5) H. Machhadani, P. Kandaswamy, S. Sakr, A. Vardi, A. Wirtmuller, L. Nevou, F. Guillot, G. Pozzovivo, M. Tchernycheva, A. Lupu, L. Vivien, P. Crozat, E. Warde, C. Bougerol, S. Schacham, G. Strasser, G. Bahir, E. Monroy, and F. H. Julien: New J. Phys. 11 (2009) 125023.

6) A. Ishida, K. Matsue, Y. Inoue, H. Fujiyasu, H.-J. Ko, A. Setiawan, J.-J. Kim, H. Makino, and T. Yao: Jpn. J. Appl. Phys. 44 (2005) 5918.

7) K. Berland, M. Stattin, R. Farivar, D. M. S. Sultan, P. Hyldgaard, A. Larsson, S. M. Wang, and T. G. Andersson: Appl. Phys. Lett. 97 (2010) 043507.

8) C. Gmachl, H. M. Ng, and A. Y. Cho: Appl. Phys. Lett. 77 (2000) 334 .

9) N. Suzuki and N. Iizuka: Jpn. J. Appl. Phys. 36 (1997) L1006.

10) F. Giorgetta, E. Baumann, F. Guillot, E. Monroy, and D. Hofstetter: Electron. Lett. 43 (2007) 185.

11) L. Nevou, M. Tchernycheva, F. H. Julien, F. Guillot, and E. Monroy: Appl. Phys. Lett. 90 (2007) 121106.

12) L. Nevou, F. H. Julien, M. Tchernycheva, F. Guillot, E. Sarigiannidou, and E. Monroy: Phys. Status Solidi C 5 (2008) 2120 .

13) M. Yamanishi, T. Edamura, K. Fujita, N. Akikusa, and H. Kan: IEEE J. Quantum Electron. 44 (2008) 12.

14) K. Driscoll, Y. Liao, A. Bhattacharyya, L. Zhou, D. J. Smith, T. D. Moustakas, and R. Paiella: Appl. Phys. Lett. 94 (2009) 081120 .

15) T. Ive, O. Brandt, H. Kostial, K. J. Friedland, L. Däweritz, and K. H. Ploog: Appl. Phys. Lett. 86 (2005) 024106.

16) M. Stattin, K. Berland, P. Hyldgaard, A. Larsson, and T. G. Andersson: Phys. Status Solidi C 8 (2011) 2357.

17) Y. Li, A. Bhattacharyya, C. Thomidis, T. D. Moustakas, and R. Paiella: Opt. Express 15 (2007) 17922.

18) C. Kumtornkittikul, T. Shimizu, N. Iizuka, N. Suzuki, M. Sugiyama, and Y. Nakano: Jpn. J. Appl. Phys. 46 (2007) L352.

19) N. Iizuka, K. Kaneko, and N. Suzuki: J. Appl. Phys. 99 (2006) 093107.

20) A. Lupu, F. Julien, S. Golka, G. Pozzovivo, G. Strasser, E. Baumann, F. Giorgetta, D. Hofstetter, S. Nicolay, M. Mosca, E. Feltin, J.-F. Carlin, and N. Grandjean: IEEE Photonics Technol. Lett. 20 (2008) 102.

21) P. Holmström: IEEE J. Quantum Electron. 42 (2006) 810.

22) M. Grundmann, H. Frenzel, A. Lajn, M. Lorenz, F. Schein, and H. von Wenckstern: Phys. Status Solidi A 207 (2010) 1437.

23) H. Y. Liu, X. Li, S. Liu, X. Ni, M. Wu, V. Avrutin, N. Izyumskaya, U. Ösgür, A. B. Yankovich, A. V. Kvit, P. M. Voyles, and H. Morkoç: Phys. Status Solidi C 8 (2011) 1548.

24) U. Özgür, Y. I. Alivov, C. Liu, A. Teke, M. A. Reshchikov, S. Dogan, V. Avrutin, S.-J. Cho, and H. Morkoc: J. Appl. Phys. 98 (2005) 041301.

25) J. Edmond, A. Abare, M. Bergman, J. Bharathan, K. L. Bunker, D. Emerson, K. Haberern, J. Ibbetson, M. Leung, P. Russel, and D. Slater: J. Cryst. Growth 272 (2004) 242.

26) H. C. Liu, M. Buchanan, and Z. R. Wasilewski: Appl. Phys. Lett. 72 (1998) 1682.

27) S. Shokhovets, R. Goldhahn, G. Gobsch, S. Piekh, R. Lantier, A. Rizzi, V. Lebedev, and W. Richter: J. Appl. Phys. 94 (2003) 307.

28) P. T. B. Shaffer: Appl. Opt. 10 (1971) 1034.

29) W. Choyke and E. Palik: in Handbook of Optical Constants of Solids, ed. E. Palik (Academic Press, New York, 1985) p. 587.

30) W.-J. Lee, J.-E. Kim, H. Y. Park, S. Park, M. su Kim, 
J. T. Kim, and J. J. Ju: J. Appl. Phys. 103 (2008) 073713.

31) G. Bentoumi, A. Deneuville, B. Beaumont, and P. Gibart: Mater. Sci. Eng. B 50 (1997) 142.

32) O. Ambacher, W. Rieger, P. Ansmann, H. Angerer, T. D. Moustakas, and M. Stutzmann: Solid State Commun. 97 (1996) 365.

33) K. Mathwig, W. Kaiser, A. Somers, J. P. Reithmaier, A. Forchel, K. Ohira, S. M. Ullah, and S. Arai: IEEE Photonics Technol. Lett. 19 (2007) 264.

34) T. Barwicz, and H. Haus: J. Lightwave Technol. 23 (2005) 2719.

35) C. Gmachl, F. Capasso, D. L. Sivco, and A. Y. Cho: Rep. Prog. Phys. 64 (2001) 1533.

36) N. Iizuka, K. Kaneko, and N. Suzuki: Appl. Phys. Lett. 81
(2002) 1803.

37) R. France, T. Xu, P. Chen, R. Chandrasekaran, and T. D. Moustakas: Appl. Phys. Lett. 90 (2007) 062115.

38) J. W. Liu, A. Kobayashi, S. Toyoda, H. Kamada, A. Kikuchi, J. Ohta, H. Fujioka, H. Kumigashira, and M. Oshima: Phys. Status Solidi B 248 (2011) 956.

39) J. W. Liu, A. Kobayashi, K. Ueno, S. Toyoda, A. Kikuchi, J. Ohta, H. Fujioka, H. Kumigashira, and M. Oshima: Appl. Phys. Lett. 97 (2010) 252111.

40) E. Kaminska, A. Piotrowska, K. Golaszewska, A. Barcz, R. Kruszka, T. Ochalski, J. Jasinski, and Z. Liliental-Weber: Phys. Status Solidi C 0 (2003) 231.

41) K. Nishizono, M. Okada, M. Kamei, D. Kikuta, K. Tominaga, Y. Ohno, and J. P. Ao: Appl. Phys. Lett. 84 (2004) 3996. 